\title{
Rebuild-up Effect on Superficial Cardiac Lesion Surrounded by Low-density Lungs: Volumetric Modulated Arc Therapy (VMAT) Planning Study Using 3-D Printed Phantom for Ventricular Tachycardia (VT)
}

\author{
Si-Won No \\ Konkuk University \\ Jun-Bong Shin \\ Asan Medical Center \\ Yong-Ki Bae \\ Konkuk University Medical Center \\ Jungmin Kim \\ Korea University \\ Semie Hong \\ Konkuk University Medical Center \\ Jeong-Woo Lee ( $\sim$ polirain@kuh.ac.kr) \\ Konkuk University Medical Center
}

\section{Research Article}

Keywords: superficial cardiac lesion, 3D (three-dimensional) printed cardiac phantom, dose rebuild-up, Gafchromic EBT3 film, stereotactic body radiation therapy (SBRT)

Posted Date: July 21 st, 2021

DOI: https://doi.org/10.21203/rs.3.rs-701961/v1

License: (c) (i) This work is licensed under a Creative Commons Attribution 4.0 International License.

Read Full License 


\section{Abstract}

The study aimed to evaluate dose distributions on the superficial cardiac lesion surrounded by lowdensity lungs. We fabricated the 3-D printed cardiac phantom to insert in a multipurpose lungman phantom (KYOTO KAGAKU, Japan) for simulating a stereotactic body radiation therapy (SBRT) in ventricular tachycardia (VT) treatment. The cardiac phantom consists of 11 slabs with $1-\mathrm{cm}$ intervals and is designed to insert radiochromic film (Gafchromic EBT3, Ashland Advanced Materials, Bridgewater, NJ) for film dosimetry. We used film dosimetry scanners (DosimetryPRO Advantage Red, Vidar Systems Corporation, Herndon, VA) with dedicated film dosimetry software (OP-IMRT, ver.1.6, IBA dosimetry, Germany). Volumetric modulated arc therapy (VMAT) technique was applied to optimize the dose distribution using the anisotropic analytic algorithm (AAA) in a radiation treatment planning (RTP) system (Eclipse v. 13.6, Varian, Palo Alto, CA). We used the 6-MV and 15-MV photon energies from a LINAC (Clinac iX, Varian, Palo Alto, CA) to investigate the planning target volume (PTV) under-dose effects due to the inner dose rebuild-up by energy dependence. The dose distributions in the VMAT plans with 6-MV and 15-MV showed good competitive coverages of the cardiac lesion without any severe underdose pattern. On the other side, the film dosimetry results showed significant dose variations near the interface of the cardiac lesion surrounded by low-density lung. The differences between the planning and the film dosimetry results revealed pretty well in both photon energies. The maximum dose differences in the cardiac PTV were ranged from $4.1-7.7 \%$ and $4.1-8.1 \%$ for $6-\mathrm{MV}$ photon beams and 15MV photon beams. Furthermore, EBT3 film measurements showed that the widths of $50 \%$ of profiles were reduced by $1.3 \mathrm{~cm}$ and $2.3 \mathrm{~cm}$ on 6-MV photon beams and 15-MV photon beams, respectively. In addition, 3-D printing techniques enabled quite challengeable dose measurements to reveal this kind of dose discrepancies in humanoid structures. This study showed that clinical cases like VT SBRT surrounded by severe inhomogeneous matter could induce wrongly to estimate appropriate dose delivery and to evaluate reasonable clinical outcomes.

\section{Introduction}

According to global cancer using the GLOBOCAN 2020 estimates of cancer incidence and mortality produced by the International Agency for Research on Cancer, about 14.1 million new cancer cases and 8.2 million deaths occurred in 2012 worldwide. Cancer is increasing due to risk factors such as smoking, overweight, physical inactivity, and changes in reproductive patterns related to urbanization and economic development and population growth, and aging ${ }^{1}$. Cancer treatment modalities comprise radiation therapy, surgery, chemotherapy, immunotherapy, and hormonal therapy. Approximately $50 \%$ of all cancer patients consider the radiation therapy process an essential component of cancer treatment, with RT contributing towards $40 \%$ of curative therapy for cancer ${ }^{2}$. RT is one of the most indispensable methods of cancer treatment in modern medicine. Stereotactic body radiotherapy (SBRT) is a newly emerging radiotherapy treatment method to deliver a high dose of radiation to the target and deliver a minimum dose to normal tissues. It utilizes either a single dose delivery or a small number of fractions with a high degree of precision within the body ${ }^{3-8}$. 
Recently, there has been clinical use of RT to treat diseases other than cancer treatment, among which cardiac SBRT is radiation therapy to help with ventricular tachycardia (VT $)^{9,10}$. Many hospitals have considered SBRT to reduce episodes of VT if VT patients fail to improve their symptoms even after performing conventional treatments, drugs, and electrode ceramics. RT in patients with VT aimed to eliminate VT symptoms, and SBRT performed high dose and single separation for heart disease. SBRT delivered in a single prescribed dose of $25 \mathrm{~Gy}$ has emerged as a new therapeutic tool in managing highly refractory $\mathrm{VT}^{11}$.

However, care is needed when treating patients with prescribed dose of $25 \mathrm{~Gy}$. Excessive exposure to lung tissue can cause radiation-induced lung injury (RILI). RILI encompasses any lung toxicity induced by RT and manifests acutely as radiation pneumonitis, bronchiolitis obliterans organizing pneumonia (BOOP) and chronically as radiation pulmonary fibrosis ${ }^{12,13}$.

Furthermore, previous studies showed different values compared to measured doses under most of the field conditions for water-equivalent, lung, rib, and hard bone densities for dose calculation algorithms in the RTP system ${ }^{14}$. Therefore, it is necessary to check the actual dose difference from the treatment planning dose distributions if the density of surrounding tissues is significantly different from that of the planning target volume (PTV), like a superficial cardiac lesion surrounded by low-density lungs.

This study aimed to evaluate the calculated dose accuracy in an RTP system compared with film dosimetry results for superficial cardiac lesions surrounded by low-density lungs using a 3-D printed cardiac phantom.

\section{Results}

We compared the calculated dose distributions from the RTP system and the measured ones by Gafchromic EBT3 film dosimetry. The calculated dose distributions using 6-MV and 15-MV beams in the RTP system showed similar coverages (Fig. 1)(Table 1). However, dose verification using the Gafchromic EBT3 film dosimetry showed a significant difference from the planned results of both beam energies.

Film dosimetry revealed a dose mismatch between the cardiac PTV and the left lung interface. In PTV, the maximum dose increased from $4.1-7.7 \%$ for 6-MV photon beams. Furthermore, EBT3 film measurements showed that $50 \%$-width of dose profiles was reduced by $1.3 \mathrm{~cm}$ on $6-\mathrm{MV}$ photon beams' plans (Fig. 2). 
Table 1

Dose statistics of target (GTVp and PTV_25) from four arcs volumetric modulated arc therapy (VMAT) plans using 6-MV and 15-MV photon beam.

\begin{tabular}{|lllllll|}
\hline Plan & Structure & Volume & Dose & Min & Max & Mean \\
& & {$\left[\mathrm{cm}^{2}\right]$} & $\begin{array}{l}\text { Cover } \\
{[\%]}\end{array}$ & $\begin{array}{l}\text { Dose } \\
{[\mathbf{c G y}]}\end{array}$ & $\begin{array}{c}\text { Dose } \\
{[\mathrm{cGy}]}\end{array}$ & $\begin{array}{c}\text { Dose } \\
{[\mathrm{cGy}]}\end{array}$ \\
\hline \multirow{2}{*}{ 6X_4Arc } & Primary Gross Tumor Volume (GTVp) & 19.4 & 100.0 & 2446.0 & 2645.4 & 2529.6 \\
\cline { 2 - 7 } & $\begin{array}{l}\text { Planning Target Volume 25Gy } \\
\text { (PTV_25) }\end{array}$ & 44.1 & 100.0 & 2241.4 & 2645.4 & 2504.8 \\
\hline 15X_4Arc & Primary Gross Tumor Volume (GTVp) & 19.4 & 100.0 & 2438.8 & 2615.3 & 2536.5 \\
\cline { 2 - 7 } & $\begin{array}{l}\text { Planning Target Volume 25Gy } \\
\text { (PTV_25) }\end{array}$ & 44.1 & 100.0 & 2244.0 & 2629.1 & 2514.6 \\
\hline
\end{tabular}

Film dosimetry results of $15 \mathrm{MV}$ beam energy also revealed a dose mismatch between the cardiac PTV and the left lung interface. In PTV, the maximum dose increased from $4.1 \%$ to $8.1 \%$ for $15-\mathrm{MV}$ photon beams. Furthermore, EBT3 film measurements showed that $50 \%$ of the treatment plan dose profiles were reduced by $2.3 \mathrm{~cm}$ on 15-MV photon beams (Fig. 3).

\section{Discussion}

When optimizing the dose distributions in commercial RTP systems, they could include some inevitable errors by hidden factors related to dose calculation algorithms. Like the superficial cardiac lesion adjacent to much low-density lung tissue, its low-density portion may affect some complex physical interactions, such as a rebuild-up effect. There are two main factors in the case of superficial cardiac lesions surrounded by low-density lungs. One is the forward-directed photon fluences due to the decreased attenuation of the beam intensity. Another thing should be the effect of electronic disequilibrium where the low-density lungs meet generic density the cardiac lesion. It may occur dose rebuild-up effect, which delivers an unwanted lower dose to the superficial cardiac lesion. These two factors are individually influenced by varying densities, with dominant factors depending on beam energy, field size, and local inhomogeneity distributions ${ }^{15}$.

Our findings would be significant because this kind of dose verification generally a very tough and challengeable trial in a humanoid phantom. Designing and fabrication of the inserted cardiac phantom was also a very delicate process for precise film dosimetry. 3-D printing technology was much beneficial for this specialized dosimetry purpose. Film dosimetry with a dedicated humanoid phantom enabled us to find a dose mismatch between the cardiac PTV and the left lung interface. The International Commission on Radiation Units and Measurements (ICRU) recommends an overall accuracy limit of $\pm 5 \%$ for dose delivery ${ }^{16}$. It says that it is crucial to maintain density-related dose uncertainty within a small range if technically achievable. It recommends setting the dose uncertainty to the smallest for tumors while maintaining steep dose-response curves and narrow therapeutic windows. 
Inhomogeneity correction performs photon dose calculations by relying on mass density or electron density information derived from CT-density conversion tables in an RTP system. Inaccurate density information can cause dose errors when non-uniformity correction is applied, and many studies have proposed tolerance levels to maintain dose errors to an acceptable extent ${ }^{17,18}$. However, more extensive changes in relative electron density, such as lung, soft palate, and bone, are significantly more expansive than the recommended tolerance values for CT number accuracy testing of diagnostic CT scanners. For this reason, if density changes are limited to within $\pm 0.02, \pm 0.03$, and $\pm 0.10 \mathrm{~g} / \mathrm{cm}^{3}$ for lung, soft tissue, and bone, respectively, the resulting dose error of the target volume will be $<2 \%$ for most clinical cases and $<3 \%$ for more challenging lung SBRT cases ${ }^{15}$. In addition, we found that it should be considered the specific irradiation conditions such as geometrical factors of inhomogeneous medium and applying beam energies as well as appropriate CT-density calibration.

Dose errors could also lessen depending on the selection of the dose calculation algorithm.

Previous studies have shown that the difference between AAA and AXB in radiotherapy plans for lung cancer is less than $3 \%$ on $\mathrm{PTV}^{19}$. Furthermore, the comparison study of AAA and AXB in individual IMRT fields showed that even though the total dose differences were minor, the dose differences for a single beam may be up to $8 \%$ in the lung interface regions. If the PTV is very close to the interface region, especially when the bone tissue is also involved, the differences between AAA and AXB might be very large $\mathrm{e}^{20}$.

We found that film dosimetry with 3-D printed phantom could be a feasible tool to perform challengeable dosimetry such as dose discrepancies of high gradient dose interface in a humanoid structure.

Dose fluctuation on the interface between superficial cardiac lesions and low-density lungs could make an error to estimate accurate dose delivery for the case of ventricular tachycardia SBRT. Also, 3-D printing techniques could be a helpful dosimetry tool to verify dose discrepancies.

\section{Methods}

\section{3-D printed cardiac phantom}

We designed a 3-D printed cardiac phantom to simulate SBRT for VT treatment. The 3-D printed cardiac phantom was designed and printed by referring to the humanoid Lungman (KYOTO KAGAKU, Japan) phantom CT images, which reproduced the pulmonary artery and the aortic arch. The size of the 3-D printed cardiac phantom is about $10-\mathrm{cm}$ in diameter in the broadest center of the heart and includes the aortic arch is about 13-cm wide, 15-cm long, and 17-cm high. The 3D design tool used the 3-Matic medical program (Mimics, Materialise, Belgium), and the 3-D printer (objet500 connex3, Stratasys, Eden Prairie, MN) was used for fabricating the phantom. It is also designing to fix in the Lungman phantom and made to resemble the human heart using Agilus and Magenta, human equivalent materials (Fig. 4). The 3-D printed cardiac phantom has also been developing to insert Gafchromic EBT3 films (Ashland, 
Bridgewater, $\mathrm{NJ}$ ), which can be separated into 11 slabs at 1-cm intervals, allowing the Gafchromic EBT3 film to settle in place after insertion.

We prepared the film by cutting to insert the 3-D printed cardiac slabs. As shown in figure 5 , the Gafchromic EBT3 film is ready for analysis across the entire heart and lungs, corresponding to the ROI (region of interest) (Fig. 5). We used the Lungman phantom, which is most similar to the human Chest shape, as shown in figure 5 so that the 3-D printed cardiac phantom with the Gafchromic EBT3 film to place inside the Lungman phantom (Fig. 5). After inserting the 3-D printed cardiac phantom, we filled the rest of the space inside the Lungman phantom with styrofoam grains, and the bottom cover of the Lungman phantom was closed to secure it.

\section{SBRT planning VT treatment and film dosimetry}

We obtained a CT image set of the Lungman phantom inserted with a 3-D cardiac phantom using a CT simulator (Large Bore, TOSHIBA, Japan). The prescribed dose scheme was $25 \mathrm{~Gy}$ in a single fraction for highly refractory ventricular tachycardia, aiming to eliminate symptoms of VT (Fig. 6). The planning technique was adopted with four arcs based volumetric modulated arc therapy (VMAT) using 6-MV and 15-MV photon beams (Table 2). Inverse planning for VMAT was applied to optimize the dose-volume basis using the anisotropic analytic algorithm (AAA) in a radiation treatment planning (RTP) system (Eclipse v. 13.6, Varian, Palo Alto, CA). We used the 6-MV and 15-MV photon energies from a LINAC (Clinac iX, Varian, Palo Alto, CA) to investigate the PTV under-dose effects due to the inner dose rebuildup by energy dependence. We used film dosimetry scanners (DosimetryPRO Advantage Red, Vidar Systems Corporation, Herndon, VA) with dedicated film dosimetry software (OP-IMRT, ver.1.6, IBA dosimetry, Germany). Finally, we analyzed the measured film as compared to the planning dose distributions from the RTP system. Figure 7 shows the schematic flow chart of the entire process of dose verification (Fig. 7).

\section{Declarations}

\section{Acknowledgments}

This work was supported by the Korea Medical Device Development Fund grant funded by the Korea government (the Ministry of Science and ICT, the Ministry of Trade, Industry and Energy, the Ministry of Health \& Welfare, the Ministry of Food and Drug Safety) (Project Number: KMDF_PR_20200901_0262)

\section{Author Contributions}

S.N. and J.S. contributed equally to this work with the first authorship. J.L. conceived the idea of this study. J.S. and S.N. designed the study under the supervising of J.L. S.N., J.S., Y.B., and S.H. performed the experiments. All authors attended reviewing and analyzed all the data. J.S., S.N., J Kim, and J.L. 
contributed to organizing this manuscript. J.L. reviewed and corrected the final manuscript with figures and tables for submission.

\section{Competing interests}

The authors declare no competing interests.

\section{Additional Information}

Correspondence and requests for materials should be addressed to J.L.

\section{Ethics approval and informed consent}

Ethics approval and formal consent do not require a phantom study.

\section{Availability of data and materials}

The datasets used and analyzed during the current study are available upon reasonable request. Please contact the authors for data requests.

\section{References}

1. Torre, L. A. et al. Global cancer statistics, 2012. CA: A Cancer Journal for Clinicians, $65,87-108$ (2015).

2. Baskar, R., Lee, K. A., Yeo, R. \& Yeoh, K. W. Cancer and Radiation Therapy: Current Advances and Future Directions. International Journal of Medical Sciences, 9, 193-199 (2012).

3. Bhattacharya, I. S. \& Hoskin, P. J. Stereotactic Body Radiotherapy for Spinal and Bone Metastases. Clin. Oncol, 27, 298-306 (2015).

4. Francolini, G. et al. Stereotactic body radiation therapy (SBRT) on renal cell carcinoma, an overview of technical aspects, biological rationale and current literature. Critical Reviews in Oncology/Hematology, 131, 24-29 (2018).

5. Ma, L., Wang, L., Tseng, C. L. \& Sahgal, A. Emerging technologies in stereotactic body radiotherapy.Chinese Clinical Oncology, 5 (2017).

6. Nagata, Y. \& Kimura, T. Stereotactic body radiotherapy (SBRT) for Stage I lung cancer. Japanese Journal of Clinical Oncology, 48, 405-409 (2018).

7. Pollom, E. L., Chin, A. L., Diehn, M., Loo, B. W. \& Chang, D. T. Normal Tissue Constraints for Abdominal and Thoracic Stereotactic Body Radiotherapy. Seminars in Radiation Oncology, 27, 197208 (2017). 
8. Potters, L. et al. American Society for Therapeutic Radiology and Oncology* and American College of Radiology Practice Guideline for the Performance of Stereotactic Body Radiation Therapy. International Journal of Radiation Oncology Biology ${ }^{*}$ Physics, 60, 1026-1032 (2004).

9. Jumeau, R. et al. [Management of refractory ventricular tachycardia using stereotactic body radiotherapy]. Rev Med Suisse, 15, 1082-1086 (2019).

10. Neuwirth, R. et al. Stereotactic radiosurgery for ablation of ventricular tachycardia. EP Europace, 21, 1088-1095 (2019).

11. Jumeau, R. et al. Tachycardies ventriculaires réfractaires: quelle place pour la radiothérapie et comment? Cancer/Radiothérapie, 24, 534-546 (2020).

12. Hanania, A. N., Mainwaring, W., Ghebre, Y. T., Hanania, N. A. \& Ludwig, M. Radiation-Induced Lung Injury: Assessment and Management. Chest, 156, 150-162 (2019).

13. Vallard, A. et al. Prévention médicale et traitement des complications pulmonaires secondaires à la radiothérapie. Cancer/Radiothérapie, 21, 411-423 (2017).

14. Alghamdi, S. \& Tajaldeen, A. Evaluation of dose calculation algorithms using different density materials for in-field and out-of-field conditions. Exp Oncol, 41, 46-52 (2019).

15. Liu, Q., Liang, J., Stanhope, C. W. \& Yan, D. The effect of density variation on photon dose calculation and its impact on intensity modulated radiotherapy and stereotactic body radiotherapy. Med. Phys, 43, 5717-5729 (2016).

16. Preface Journal of the International Commission on Radiation Units and Measurements, 14, 1-1 (2014).

17. Chu, J. C. H., Ni, B., Kriz, R., Amod, V. \& Saxena Applications of simulator computed tomography number for photon dose calculations during radiotherapy treatment planning. Radiotherapy and Oncology, 55, 65-73 (2000).

18. Thomas, S. J. Relative electron density calibration of CT scanners for radiotherapy treatment planning. Br J Radiol, 72, 781-786 (1999).

19. Fogliata, A., Nicolini, G., Clivio, A., Vanetti, E. \& Cozzi, L. Critical Appraisal of Acuros XB and Anisotropic Analytic Algorithm Dose Calculation in Advanced Non-Small-Cell Lung Cancer Treatments. International Journal of Radiation Oncology*Biology*Physics, 83, 1587-1595 (2012).

20. Han, T. et al. Dosimetric impact of Acuros XB deterministic radiation transport algorithm for heterogeneous dose calculation in lung cancer. Medical physics, 40, 051710-051710 (2013).

\section{Figures}





Figure 1

Results of volumetric modulation arc therapy (VMAT) plans using 6-MV and 15-MV photon beams.

(a)
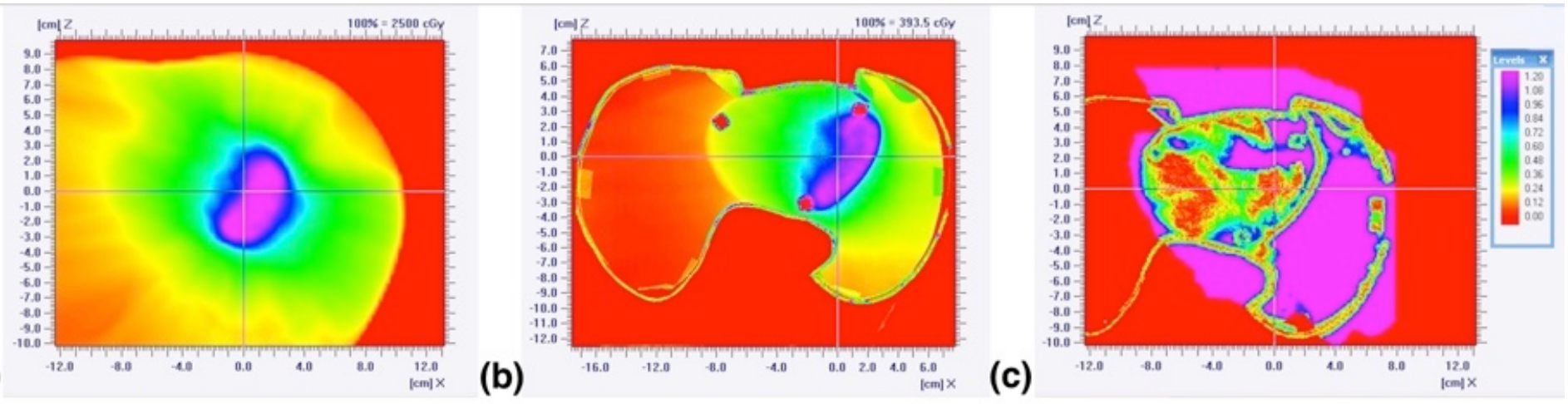

$[\%]$ Signal

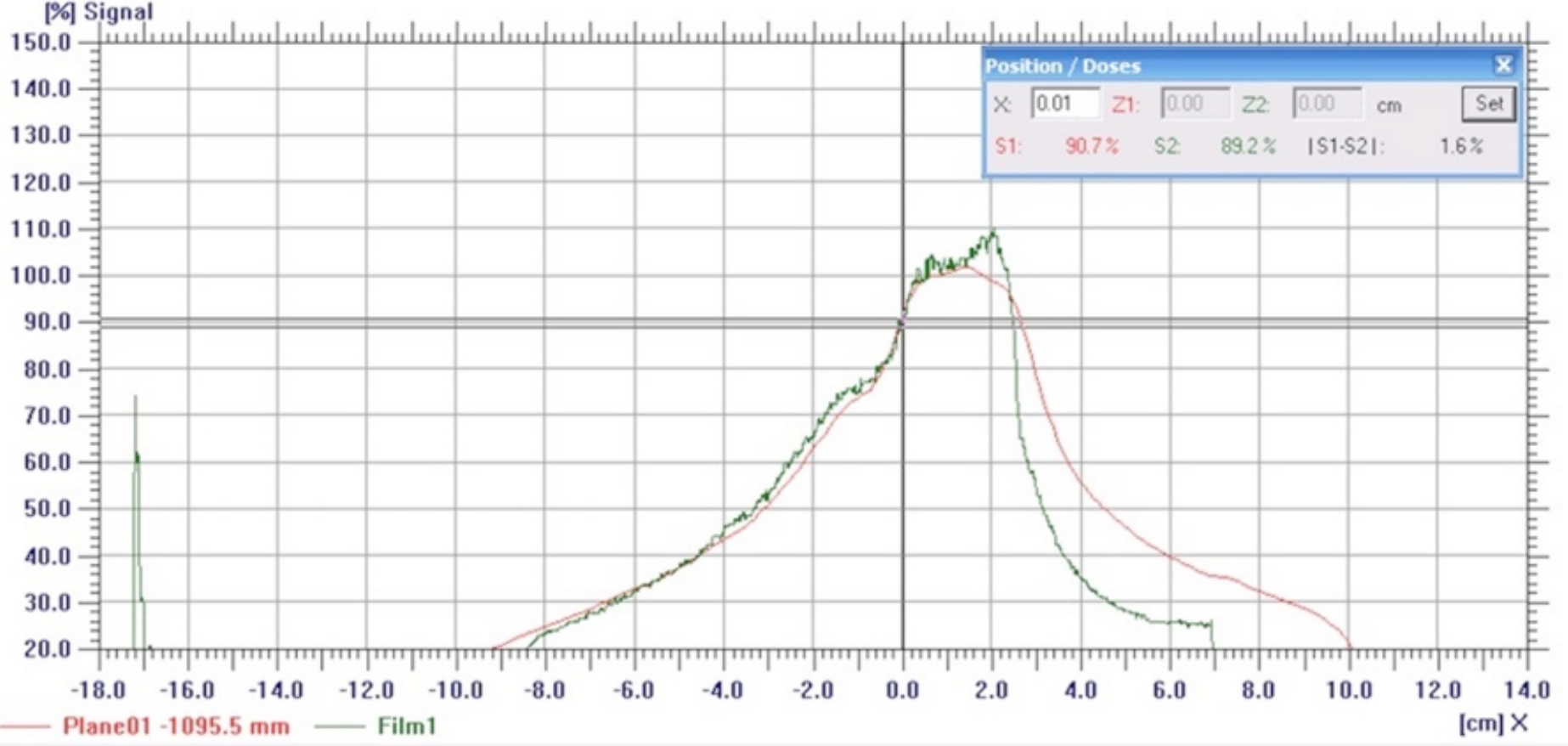

Figure 2 
Dose profile analysis of film measurement (EBT3) versus treatment plan from 6-MV VMAT paln. (a) planned dose, (b) measured dose, and (c) dose profiles comparison.

(a)
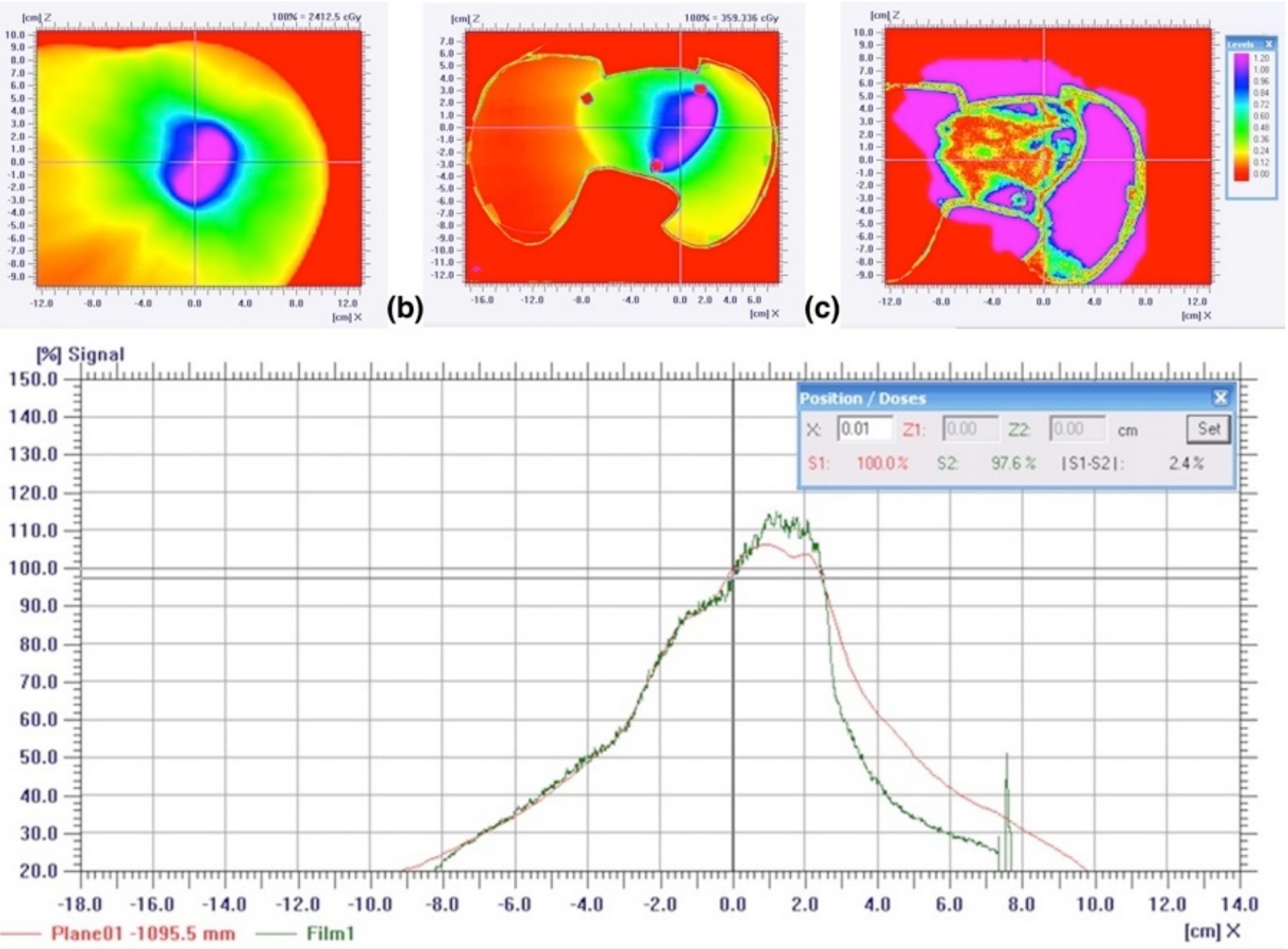

\section{Figure 3}

Dose profile analysis of film measurement (EBT3) versus treatment plan from 15-MV VMAT paln. (a) planned dose, (b) measured dose, and (c) dose profiles comparison

(a)
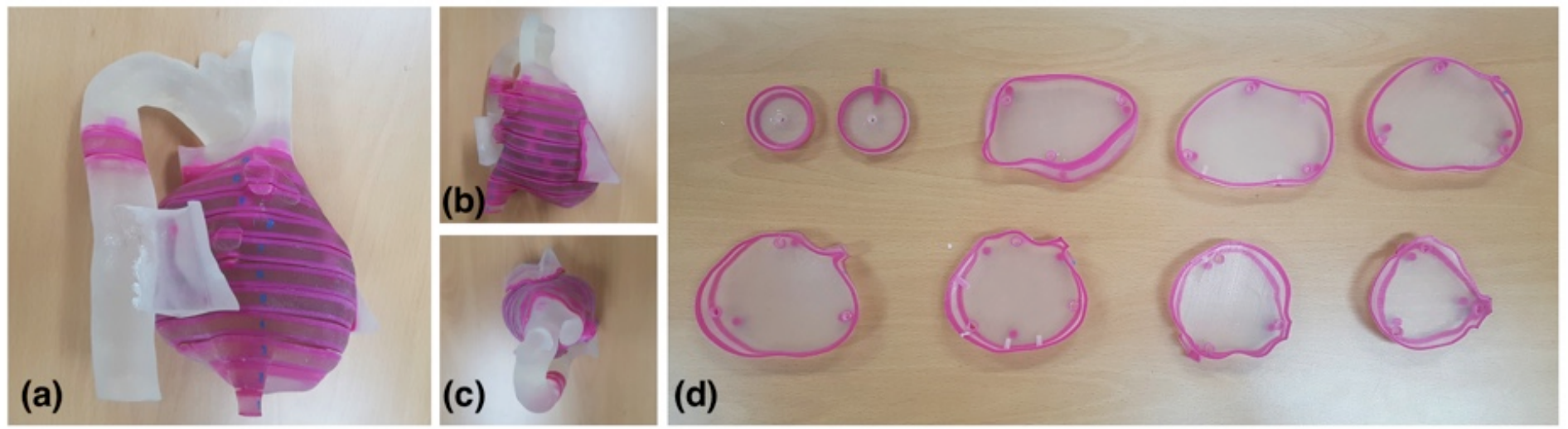

Figure 4 
3-D printed cardiac phantom set. (a) lateral view, (b) anterior view, (c) top-down view, and (d) cardiac phantom slabs

(a)

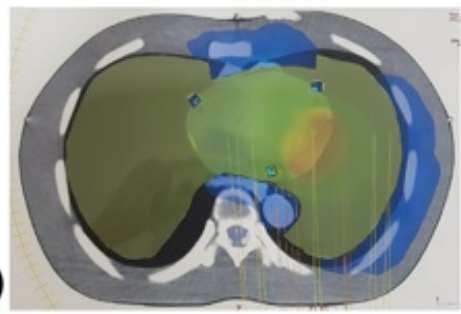

(b)

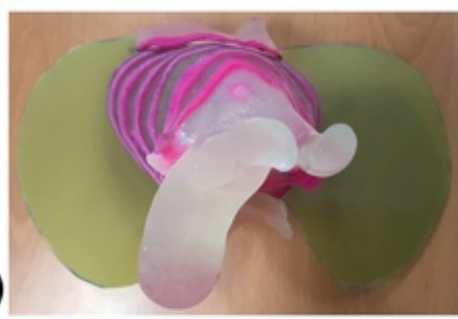

(c)

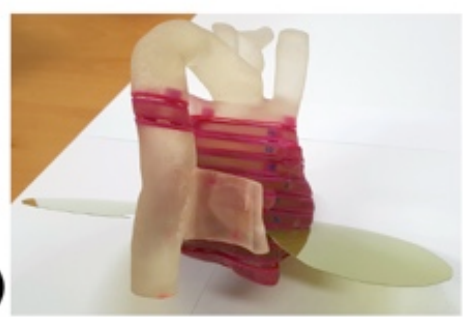

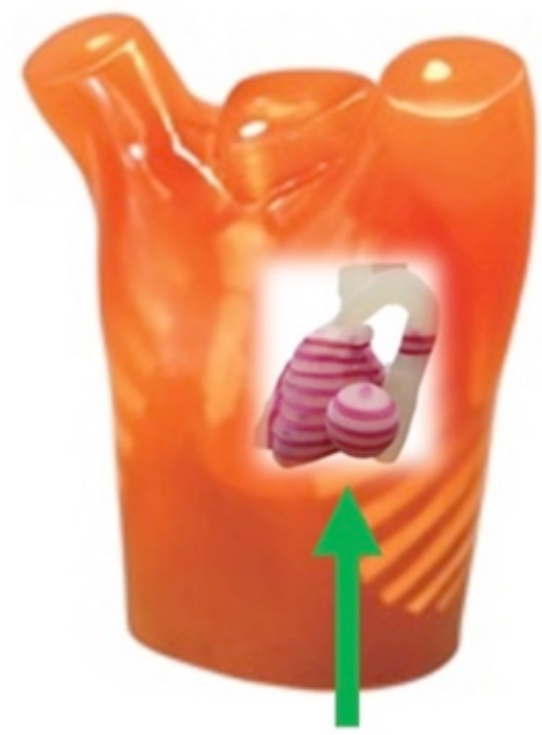

(d)

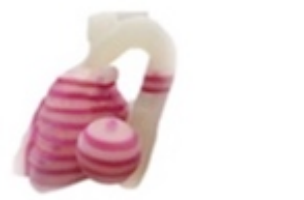

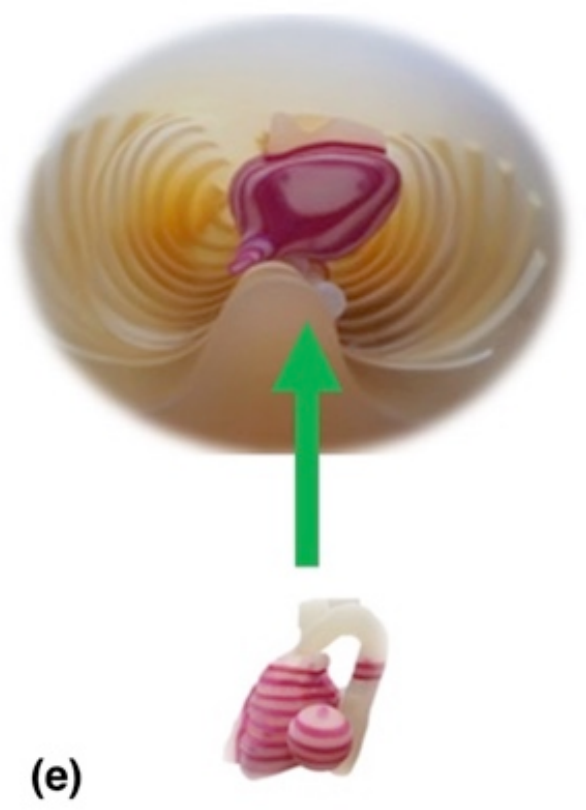

(e)

Figure 5

Film dosimetry set-up with 3D printed cardiac phantoms. (a) axial view, (b) top-down view, (c) lateral view of 3-D cardiac phantom, and (d) anterio-oblique view, (e) bottom-up view of the Lungman phantom inserted 3D printed cardiac phantom. 


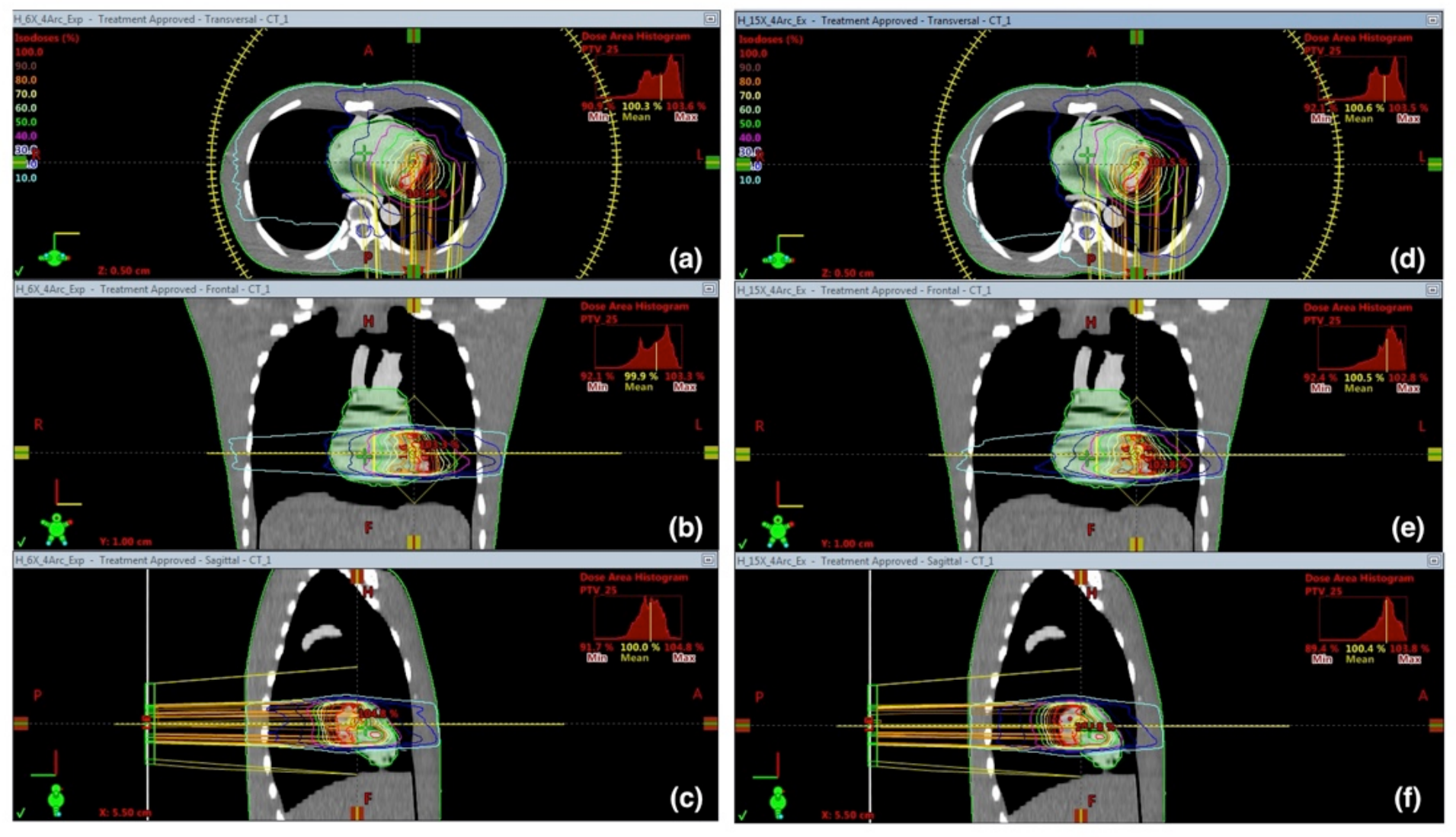

Figure 6

Dose distributions from VMAT plans using 6-MV and 15-MV photon beam. (a) axial view, (b) coronal view, (c) sagittal view from 6-MV VMAT plan, and (d) axial view, (e) coronal view, (f) sagittal view from 15-MV VMAT plan. 


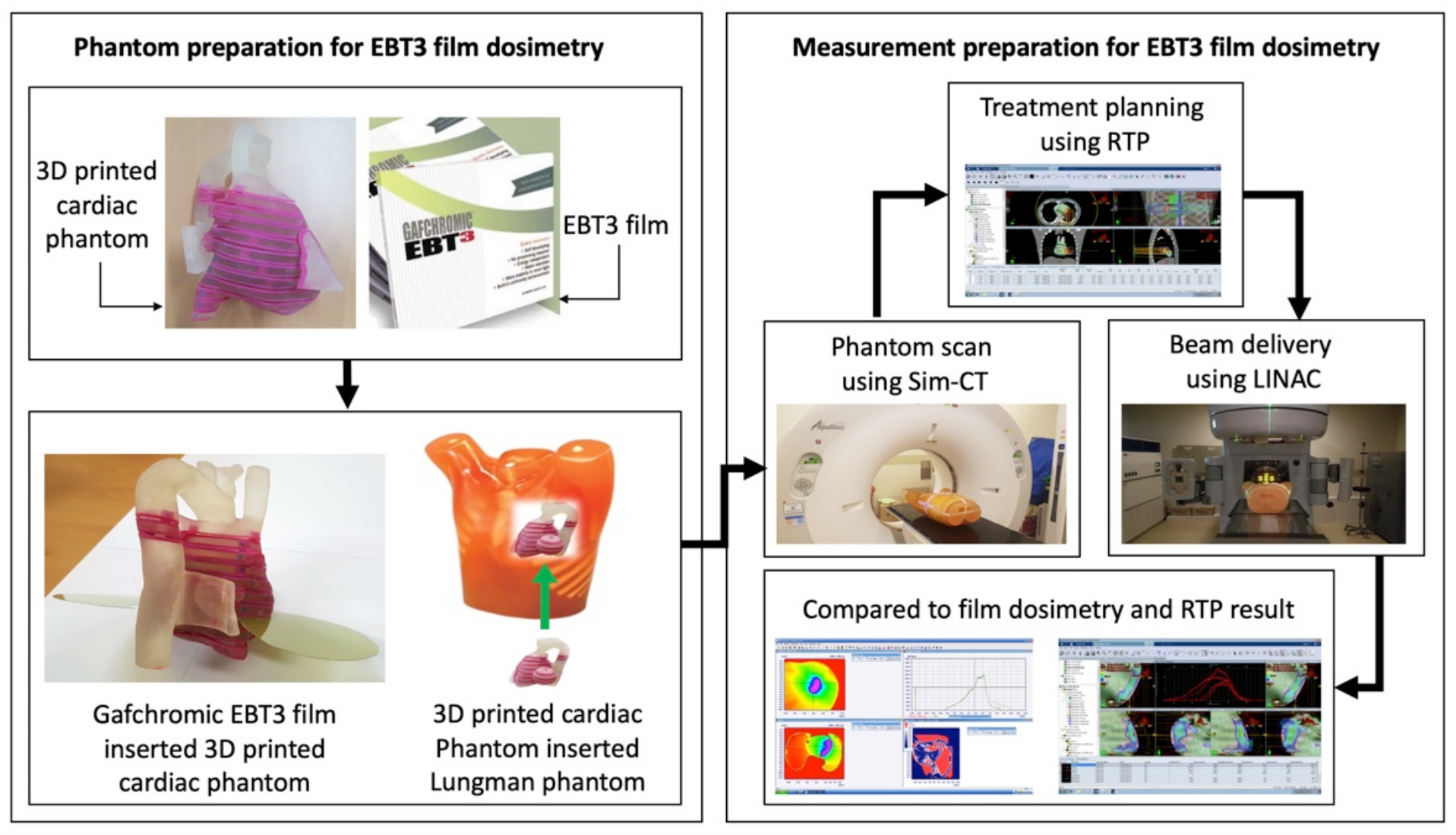

Figure 7

Overall procedures of the experiments and analysis for the study. 